\title{
Seleção de linhagens de Trichogramma (Hymenoptera, Trichogrammatidae) e determinação do número de parasitóides a ser liberado para o controle de Gymnandrosoma aurantianum Lima (Lepidoptera, Tortricidae)
}

\author{
Rosa Maria da Silva Molina \& José Roberto Postali Parra
}

Departamento de Entomologia, Fitopatologia e Zoologia Agrícola, Escola Superior de Agricultura "Luiz de Queiroz", Universidade de São Paulo. Avenida Pádua Dias, 11, Caixa Postal 9, 13418-900 Piracicaba-SP, Brasil.rmsmolina@ig.com.br; jrpparra@esalq.usp.br

\begin{abstract}
Selection of Trichogramma strains and determination of number of parasitoids to be released to control Gymnandrosoma aurantianum Lima (Lepidoptera, Tortricidae). In order to select Trichogramma strains to control $G$. aurantianum, the biological characteristics of 13 Trichogramma species/strains were evaluated. After selection, the ideal number of T. pretiosum (G18 strain) to be released per G. aurantianum egg was determined in greenhouse tests. The selection test for species/strains was carried out in an incubator adjusted to $25 \pm 1{ }^{\circ} \mathrm{C}, \mathrm{RH} 70 \pm 10 \%$, and a $14 \mathrm{~h}$ photophase. The ideal number of parasitoids was estimated in cages covered with a piece of voile-type fabric. The cycle duration for the 13 Trichogramma species/strains varied from 10.2 to 11.9 days. The Atp strain (T. atopovirilia) showed greater parasitism capacity, with an average of 23.3 parasitized eggs and $77.5 \%$ parasitism in 24 hours. The G18 strain ( $T$. pretiosum) came next, with an average of 16.8 parasitized eggs and $56.1 \%$ parasitism during the same time interval. The emergency, longevity of males, and sex ratio for the 13 species/strains were similar. The number of adults emerged per egg was 1.8 for strains G11 (T. pretiosum) and Br10 (T. bruni), which were different from the G3 strain (T. pretiosum) only, with 1.3 adults per egg. With regard to female longevity, distinct values were observed only between $T$. pretiosum strains $\mathrm{Tp}$ and $\mathrm{L} 2$, with 6.3 and 9.3 days, respectively. Under greenhouse conditions, the estimated ratio of $36 T$. pretiosum parasitoids per G. aurantianum egg allowed the highest percentage of parasitism (89\%). Therefore, Trichogramma spp. has a potential to control G. aurantianum, as long as a large number of parasitoids is released per unit area.
\end{abstract}

KEYWORDS. Applied biological control; Citrus; citrus fruit borer; egg parasitoids.

RESUMO. Seleção de linhagens de Trichogramma e determinação do número de parasitóides a ser liberado para o controle de Gymnandrosoma aurantianum Lima (Lepidoptera, Tortricidae). Com o objetivo de selecionar linhagens de Trichogramma para o controle de G. aurantianum, avaliaram-se as características biológicas de 13 linhagens/espécies deste parasitóide. A partir desta seleção, determinou-se o número ideal de T. pretiosum (linhagem G18) a ser liberado por ovo de G. aurantianum em testes de telado. O teste de seleção de linhagens/espécies foi realizado em câmara climatizada regulada a $25 \pm 1^{\circ} \mathrm{C}$, UR: $70 \pm 10 \%$ e fotofase de $14 \mathrm{~h}$. O número ideal de parasitóides foi estimado em gaiolas recobertas com tecido do tipo "voile". A duração do ciclo de vida das 13 linhagens/espécies de Trichogramma variou de 10,2 a 11,9 dias. A linhagem Atp (T. atopovirilia) apresentou maior capacidade de parasitismo, com uma média de 23,3 ovos parasitados e 77,5\% de parasitismo em 24 horas, vindo a seguir a linhagem G18 (T. pretiosum) com média de 16,8 ovos parasitados e 56,1\% de parasitismo no mesmo intervalo de tempo. As 13 linhagens/espécies tiveram desempenho semelhante quanto à emergência, longevidade de machos e razão sexual. O número de adultos emergidos por ovo foi de 1,8 para as linhagens G11 (T. pretiosum) e Br10 (T. bruni), as quais diferiram apenas da linhagem G3 (T. pretiosum), esta com 1,3 indivíduo/ovo. Para a longevidade de fêmeas foram encontrados valores distintos apenas entre as linhagens de T. pretiosum Tp e L2, com 6,3 e 9,3 dias, respectivamente. A proporção estimada de 36 parasitóides por ovo de $G$. aurantianum possibilitou a maior porcentagem de parasitismo por T. pretiosum (89\%) nas condições de telado. Portanto, Trichogramma spp. apresentam potencial de controle de G. aurantianum, desde que liberados em grandes quantidades por unidade de área.

PALAVRAS-CHAVE. Bicho-furão; Citrus; controle biológico aplicado; parasitóides de ovos.

Para o sucesso de um programa de controle biológico é importante a escolha adequada de uma espécie ou linhagem de inimigo natural a ser utilizada. O grande número de espécies, e/ou linhagens de Trichogramma (Hymenoptera, Trichogrammatidae), criadas "in vivo" ou "in vitro", apresentam diferenças que podem alterar sua eficiência no controle de uma determinada praga. Segundo Hassan (1989), essas diferenças variam em relação à sua preferência por hospedeiros, culturas (reconhecimento e aceitação), no comportamento de busca (localização do hábitat e hospedeiro) e na tolerância às condições ambientais.
Vários estudos têm demonstrado que sucessos e insucessos no uso destes parasitóides dependerão, dentre outros fatores, da escolha da espécie, ou mesmo linhagem, e por isso tornam-se importantes os estudos de seleção de linhagens e/ou espécies para obtenção de parasitóides mais eficientes, melhor adaptados à cultura e/ou hospedeiro e às diferentes condições climáticas (Hassan 1997).

Resultados promissores foram encontrados em pesquisas com Trichogramma spp. para o controle de Gymnandrosoma aurantianum Lima, 1927 (Lepidoptera Tortricidae), importante praga da citricultura paulista, que tem causado perdas elevadas 
(Garcia 1998; Garcia \& Parra 1999) e que correspondem a um prejuízo de cerca de 50 milhões de dólares por ano à citricultura paulista (Fundecitrus 2000).

Com o objetivo de avaliar o potencial de utilização de linhagens/espécies de Trichogramma, como mais um componente do manejo de G. aurantianum, foram realizadas avaliações biológicas envolvendo seleção de linhagens e determinação do número ideal de parasitóides a ser liberado por ovo da praga em condições de telado.

\section{MATERIALE MÉTODOS}

Criação dos hospedeiros e parasitóides. Os ovos de $G$. aurantianum e Anagasta kuehniella (Zeller, 1879) (Lepidoptera, Pyralidae) utilizados nos experimentos foram obtidos das criações mantidas no Laboratório de Biologia de Insetos do Departamento de Entomologia, Fitopatologia e Zoologia Agrícola, da Escola Superior de Agricultura "Luiz de Queiroz”, Universidade de São Paulo (ESALQ/USP). As linhagens/espécies de Trichogramma foram criadas e multiplicadas em ovos de A. kuehniella. As criações foram conduzidas segundo as metodologias de Parra (1997), para a traça e o parasitóide, e de Garcia \& Parra (1999) para G. aurantianum.

Seleção de linhagens/espécies de Trichogramma. Dentre as linhagens estudadas, as 10 linhagens de Trichogramma pretiosum Riley, 1879 (Hymenoptera, Trichogrammatidae) (identificadas como G1, G2, G3, G4, G11, G18, L2, L3, L4 e Tp) foram coletadas em ovos de Spodoptera frugiperda (J. E. Smith, 1797) (Lepidoptera, Noctuidae), em plantios de milho, em Piracicaba-SP. As duas linhagens de Trichogramma bruni Nagaraja, 1983 (identificadas como Br1 e Br10) foram coletadas em ovos de Heliconius erato phyllis (Fabricius, 1775) (Lepidoptera, Nymphalidae), em Piracicaba-SP e a de Trichogramma atopovirilia Oatman \& Platner, 1983 (identificada como Atp) foi fornecida pela Embrapa Semi-Árido, Petrolina-PE. Todas as linhagens utilizadas pertencem à coleção do Departamento de Entomologia, Fitopatologia e Zoologia Agrícola da ESALQ/USP de Piracicaba-SP.

Para a seleção das linhagens/espécies sobre ovos de $G$. aurantianum foram individualizadas fêmeas de cada linhagem, com 24 horas de idade. Estas foram colocadas em tubos de vidro $(12 \times 75 \mathrm{~mm})$ tampados com filme plástico de PVC (Magipack ${ }^{\circledR}$ ) e alimentadas com uma gotícula de mel puro. Para cada fêmea foram oferecidos 30 ovos de G. aurantianum, com no máximo 24 horas de desenvolvimento embrionário. $\mathrm{O}$ parasitismo foi permitido por 24 horas e, então, as fêmeas foram retiradas dos tubos de vidro e, nestes, foram colocados chumaços de algodão, umedecidos com sulfato de cobre a $1 \%$, para evitar ressecamento e o crescimento de fungos nos ovos, sendo em seguida tampados com algodão hidrófilo. Cada linhagem (tratamento) foi representada por cinco repetições, sendo cada repetição constituída por uma parcela com cinco tubos. Os experimentos foram instalados em câmaras climatizadas reguladas a $25 \pm 1{ }^{\circ} \mathrm{C}$, UR: $70 \pm 10 \%$ e fotofase de 14 horas.

Avaliaram-se os parâmetros biológicos: duração do ciclo (ovo-adulto), número de ovos parasitados, porcentagem de parasitismo, porcentagem de emergência, número de adultos emergidos por ovo, longevidade de fêmeas e machos e razão sexual.

Os dados referentes aos parâmetros biológicos foram submetidos à análise de variância e as médias comparadas, pelo teste de Tukey $(\mathrm{P} \leq 0,05)$, utilizando-se o programa SANEST (Sistema de Análise Estatística). Para razão sexual, os dados foram transformados em $\log (\mathrm{x}+1)$ e a porcentagem de parasitismo e de emergência para arc sen $\sqrt{(\mathrm{x} / 100)}$.

Determinação do número ideal de parasitóides ser liberado por ovo de G aurantianum. Foi estimado por meio de liberações de números variáveis de $T$. pretiosum (linhagem G18), em relação a um número fixo de ovos de G. aurantianum. Os ovos foram obtidos dos substratos de postura da criação do bichofurão do Laboratório de Biologia de Insetos da ESALQ/USP e foram expostos ao parasitismo no interior de gaiolas com armação de ferro $(40 \times 40 \times 120 \mathrm{~cm})$, recobertas com tecido branco e fino ("voile"). No interior destas gaiolas foi colocada uma muda de citros da variedade Valência, com cerca de dois anos de idade, para a fixação dos ovos de G. aurantianum. Os ovos foram distribuídos pela planta, em substrato de postura (pedaços de material plástico), contendo de um a dois ovos, sendo estes recortados e fixados às plantas (em toda a sua extensão) com fita adesiva. O número de ovos foi baseado no que ocorre na natureza (Garcia 1998).

Foram utilizados 30 ovos de bicho-furão por planta, sendo liberadas 48, 96, 192, 384, 768 e 1536 fêmeas de T. pretiosum, ou seja, nas proporções de 1,$6 ; 3,2 ; 6,4 ; 12,8 ; 25,6$ e 51,2 parasitóides por ovo de G. aurantianum, num total de seis tratamentos, em delineamento experimental inteiramente casualizado. Estes estudos foram conduzidos em telado, sem controle de temperatura, umidade relativa e luz. O experimento foi realizado em duas épocas, sendo a primeira em 16/03/2002 e a segunda em 27/03/2002. Na primeira época foram utilizadas quatro repetições, sendo acrescentado um tratamento extra, sem a liberação dos parasitóides, o qual não foi incluído na análise estatística e serviu como testemunha para verificar a eficiência de isolamento das gaiolas em impedir a passagem de parasitóides de uma gaiola para outra. Como não houve parasitismo na testemunha, na segunda época não foi incluído aquele tratamento e o número de repetições foi aumentado para cinco.

O parasitismo foi permitido por 24 horas, quando os ovos foram então recolhidos e mantidos em placas de Petri a $25^{\circ} \mathrm{C}$ até o seu "escurecimento", para avaliação da porcentagem de parasitismo.

Os dados de porcentagem de parasitismo foram transformados em arc sen $\sqrt{(\mathrm{x} / 100)}$ e submetidos à análise de variância conjunta dos experimentos e à análise de regressão, utilizando-se o programa SAS (SAS Institute 1999). 


\section{RESULTADOSEDISCUSSÃO}

Seleção de linhagens/espécies. A duração do período ovoadulto das treze linhagens/espécies de Trichogramma em ovos de G. aurantianum variou de 10,2 a 11,9 dias. A menor duração foi observada na linhagem $\mathrm{G} 1$, a qual não diferiu significativamente das linhagens G18, L3, G11, G2, G3 e G4 (Tabela I).

Os períodos de desenvolvimento, embora tenham diferido entre algumas linhagens/espécies, foram próximos aos observados por Beserra (2000), para 20 linhagens de $T$. pretiosum em ovos de $S$. frugiperda (9,6 a 10,1 dias), e por Pratissoli \& Parra (2001) para seis linhagens de T. pretiosum em ovos de Tuta absoluta (Meyrick, 1917) (Lepidoptera, Noctuidae) e Phthorimaea operculella (Zeller, 1873) (Lepidoptera, Gelechiidae) (9,6 a 11,5); entretanto, foram maiores do que os valores observados por Beserra \& Parra (2004) para T. pretiosum e T. atopovirilia em ovos de $S$. frugiperda $(9,6$ e 9,8); e, menores que os observados por Sá \& Parra (1994) para duas linhagens de T. pretiosum em ovos de A. kuehniella $(13,1)$.

A linhagem Atp apresentou maior capacidade de parasitismo (número médio de ovos parasitados e porcentagem de parasitismo), observando-se uma média de 23,3 ovos parasitados e $77,5 \%$ de parasitismo em 24 horas, diferindo significativamente das demais linhagens/espécies avaliadas (Tabela I). A linhagem G18 apresentou o segundo maior valor para estas características, com média de 16,8 ovos parasitados e $56,1 \%$ de parasitismo, não diferindo das linhagens G1, G3, L3, L4 quanto ao número de ovos parasitados, e das linhagens G1, G3 L3, L4, Tp e G2, com relação à porcentagem de parasitismo (Tabela I).

Diferenças no potencial de parasitismo entre linhagens e/ ou espécies de Trichogramma têm sido relatadas em diversas pesquisas (Hassan 1989; Hassan \& Guo 1991; Wührer \&
Hassan 1993; Li et al. 1994; Torres \& Gerding 2000; Pratissoli et al. 2004).

As treze linhagens/espécies estudadas tiveram desempenho semelhante quanto à emergência, longevidade de machos e razão sexual, não diferindo estatisticamente entre si (Tabelas I e II).

A porcentagem de emergência das linhagens/espécies de Trichogramma criadas sobre ovos de G. aurantianum a $25^{\circ} \mathrm{C}$ foi inferior àquela relatada por outros pesquisadores para estes parasitóides em diferentes hospedeiros, pois a literatura relata uma faixa de emergência variável de 68,7 a 100\% (Calvin et al. 1984; Harrison et al. 1985; Bleicher \& Parra 1989; Pratissoli \& Parra 2000; Beserra \& Parra 2004; Pratissoli et al. 2004). A baixa porcentagem de emergência observada em todos os experimentos poderia estar ligada à dificuldade de manutenção da umidade no interior dos tubos para evitar o ressecamento dos ovos de G. aurantianum. Embora a umidade relativa na câmara climatizada tenha variado entre $70 \pm 10 \%$ e os tubos contivessem chumaços de algodão umedecido no seu interior, ainda assim observou-se que os ovos de G. aurantianum ressecaram. Segundo Garcia (1998), os ovos de G. aurantianum não são tão suscetíveis à dessecação, pois há eclosão semelhante de lagartas a 50, 70 e $90 \%$ de umidade relativa; entretanto, no presente trabalho observou-se dificuldade dos adultos para romperem o córion e, quando emergiu mais de um adulto por ovo, observou-se redução no tamanho dos mesmos (baseando-se apenas em observações visuais).

Vários autores têm encontrado diferenças na aceitação de diferentes hospedeiros para parasitismo (Hassan \& Guo 1991; Wührer \& Hassan 1993; Torres \& Gerding 2000; Pratissoli \& Parra 2001; Pratissoli et al. 2004). Segundo Hassan (1997), linhagens de diferentes agroecossistemas podem apresentar diferenças quanto ao seu potencial. A menor aceitação (parasitismo) de ovos de G. aurantianum, observada no presente trabalho, pode estar ligada ao fato de que os

Tabela I. Duração do período ovo-adulto, número de ovos parasitados, porcentagem de parasitismo e porcentagem de emergência de 13 linhagens/ espécies de Trichogramma criadas em ovos de Gymnandrosoma aurantianum. Período de parasitismo: 24 horas; temperatura: $25 \pm 2^{\circ} \mathrm{C}$; UR: 70 $\pm 10 \%$ e fotofase: 14 horas.

\begin{tabular}{|c|c|c|c|c|c|}
\hline $\begin{array}{l}\text { Linhagem/ } \\
\text { Espécie }\end{array}$ & $\begin{array}{l}\text { Duração (ovo- } \\
\text { adulto) (dias) }\end{array}$ & $\begin{array}{c}\text { Número de } \\
\text { ovos parasitados }\end{array}$ & Parasitismo(\%) & Emergência(\% & \\
\hline Atp & $11,4 \pm 0,33$ abc & $23,3 \pm 2,12 \mathrm{a}$ & $77,5 \pm 4,09$ a & $30,9 \pm 5,62$ & $\mathrm{a}$ \\
\hline G18 & $10,4 \pm 0,16 \quad \mathrm{~cd}$ & $16,8 \pm 1,89 \mathrm{~b}$ & $56,1 \pm 6,55 \mathrm{~b}$ & $42,1 \pm 9,82$ & $\mathrm{a}$ \\
\hline G1 & $10,2 \pm 0,24$ & $16,0 \pm 1,22 b c$ & $53,3 \pm 8,32$ & $35,7 \pm 10,77$ & $\mathrm{a}$ \\
\hline G3 & $10,9 \pm 0,23$ abcd & $15,6 \pm 1,89 \quad \mathrm{bc}$ & $51,6 \pm 12,77 \quad b$ & $42,0 \pm 5,14$ & $\mathrm{a}$ \\
\hline L3 & $10,7 \pm 0,29 \quad$ bcd & $13,2 \pm 3,83$ bcd & $43,5 \pm 9,27 \quad b c$ & $39,6 \pm 6,50$ & $\mathrm{a}$ \\
\hline $\mathrm{L} 4$ & $11,5 \pm 0,11 \mathrm{ab}$ & $12,8 \pm 1,33 \mathrm{bcd}$ & $42,3 \pm 4,46 \quad b c$ & $42,6 \pm 5,93$ & a \\
\hline $\mathrm{Tp}$ & $11,9 \pm 1,41$ a & $11,8 \pm 2,78 \quad$ cde & $39,3 \pm 6,30$ & $37,6 \pm 8,77$ & $\mathrm{a}$ \\
\hline $\mathrm{G} 2$ & $10,9 \pm 0,21 \mathrm{abcd}$ & $11,2 \pm 1,23$ & $38,0 \pm 6,31$ & $40,6 \pm 10,46$ & $\mathrm{a}$ \\
\hline G11 & $10,8 \pm 0,32$ abcd & $9,0 \pm 2,21$ & $30,4 \pm 7,08$ & $40,6 \pm 4,91$ & $\mathrm{a}$ \\
\hline Br1 & $11,7 \pm 0,32 \mathrm{ab}$ & $8,4 \pm 2,49$ & $27,7 \pm 4,12$ & $36,9 \pm 11,35$ & $\mathrm{a}$ \\
\hline G4 & $10,9 \pm 0,41$ abcd & $7,2 \pm 1,96$ & $23,9 \pm 3,41$ & $37,9 \pm 15,72$ & $\mathrm{a}$ \\
\hline Br10 & $11,6 \pm 0,11 a b$ & $6,2 \pm 3,50$ & $20,8 \pm 7,36$ & $36,2 \pm 10,00$ & $\mathrm{a}$ \\
\hline $\mathrm{L} 2$ & $11,4 \pm 0,51 \mathrm{abc}$ & $5,0 \pm 1,02$ & $16,0 \pm 11,68$ & $38,9 \pm 9,12$ & $\mathrm{a}$ \\
\hline
\end{tabular}

Atp = T. atopovirilia $; \mathrm{G}, \mathrm{T}, \mathrm{L}=$ linhagens de T. pretiosum; $\mathrm{Br} 1$ e $\mathrm{Br} 10=$ linhagens de T. bruni.

Médias seguidas da mesma letra, na coluna, não diferem significativamente entre si, pelo teste de Tukey, ao nível de 5\% de probabilidade. 
Tabela II. Número de adultos emergidos por ovo, longevidade de fêmeas e machos e razão sexual de 13 linhagens/espécies de Trichogramma criadas em ovos de Gymnandrosoma aurantianum. Período de parasitismo: 24 horas; temperatura: $25 \pm 2^{\circ} \mathrm{C}$; UR: $70 \pm 10 \%$ e fotofase: 14 horas.

\begin{tabular}{llccc}
\hline $\begin{array}{l}\text { Linhagem/ } \\
\text { Espécie }\end{array}$ & $\begin{array}{c}\text { Número de } \\
\text { adultos por ovo }\end{array}$ & $\begin{array}{c}\text { Longevidade de fêmeas } \\
\text { (dias) }\end{array}$ & $\begin{array}{c}\text { Longevidade de } \\
\text { machos (dias) }\end{array}$ & $\begin{array}{c}\text { Razão } \\
\text { sexual }\end{array}$ \\
\hline Atp & $1,5 \pm 0,08 \mathrm{ab}$ & $7,0 \pm 0,28 \mathrm{ab}$ & $6,5 \pm 0,58 \mathrm{a}$ & $0,8 \pm 0,04 \mathrm{a}$ \\
$\mathrm{G} 18$ & $1,4 \pm 0,11 \mathrm{ab}$ & $7,9 \pm 0,36 \mathrm{ab}$ & $5,6 \pm 1,02 \mathrm{a}$ & $0,8 \pm 0,05 \mathrm{a}$ \\
$\mathrm{G} 1$ & $1,4 \pm 0,10 \mathrm{ab}$ & $6,5 \pm 1,35 \mathrm{ab}$ & $6,0 \pm 0,76 \mathrm{a}$ & $0,8 \pm 0,05 \mathrm{a}$ \\
$\mathrm{G} 3$ & $1,3 \pm 0,20 \mathrm{~b}$ & $8,9 \pm 1,02 \mathrm{ab}$ & $6,4 \pm 0,66 \mathrm{a}$ & $0,7 \pm 0,14 \mathrm{a}$ \\
L3 & $1,4 \pm 0,11 \mathrm{ab}$ & $8,5 \pm 1,98 \mathrm{ab}$ & $6,1 \pm 1,20 \mathrm{a}$ & $0,8 \pm 0,04 \mathrm{a}$ \\
L4 & $1,6 \pm 0,20 \mathrm{ab}$ & $8,4 \pm 1,15 \mathrm{ab}$ & $6,2 \pm 0,62 \mathrm{a}$ & $0,7 \pm 0,10 \mathrm{a}$ \\
Tp & $1,7 \pm 0,08 \mathrm{ab}$ & $6,3 \pm 1,24 \mathrm{~b}$ & $6,5 \pm 0,56 \mathrm{a}$ & $0,8 \pm 0,10 \mathrm{a}$ \\
G2 & $1,4 \pm 0,45 \mathrm{ab}$ & $8,6 \pm 1,71 \mathrm{ab}$ & $6,5 \pm 0,72 \mathrm{a}$ & $0,7 \pm 0,11 \mathrm{a}$ \\
G11 & $1,8 \pm 0,15 \mathrm{a}$ & $7,1 \pm 0,85 \mathrm{ab}$ & $6,8 \pm 0,37 \mathrm{a}$ & $0,7 \pm 0,08 \mathrm{a}$ \\
Br1 & $1,4 \pm 0,19 \mathrm{ab}$ & $6,9 \pm 1,39 \mathrm{ab}$ & $6,0 \pm 1,60 \mathrm{a}$ & $0,8 \pm 0,05 \mathrm{a}$ \\
G4 & $1,6 \pm 0,24 \mathrm{ab}$ & $8,4 \pm 2,37 \mathrm{ab}$ & $5,6 \pm 0,47 \mathrm{a}$ & $0,8 \pm 0,07 \mathrm{a}$ \\
Br10 & $1,8 \pm 0,32 \mathrm{a}$ & $6,7 \pm 1,50 \mathrm{ab}$ & $6,0 \pm 1,73 \mathrm{a}$ & $0,8 \pm 0,05 \mathrm{a}$ \\
L2 & $1,7 \pm 0,38 \mathrm{ab}$ & $9,3 \pm 0,80 \mathrm{a}$ & $5,6 \pm 1,06 \mathrm{a}$ & $0,6 \pm 0,46 \mathrm{a}$ \\
\hline
\end{tabular}

Atp = T. atopovirilia $; \mathrm{G}, \mathrm{T}, \mathrm{L}=$ linhagens de T. pretiosum; $\mathrm{Br} 1$ e $\mathrm{Br} 10=$ linhagens de T. bruni .

Médias seguidas da mesma letra, na coluna, não diferem significativamente entre si, pelo teste de Tukey, ao nível de 5\% de probabilidade.

parasitóides não se desenvolveram, em nenhuma geração, no ovo da praga e, principalmente, por não ser o bicho-furão o hospedeiro de origem de nenhuma delas. Com base na presente pesquisa, a manutenção da linhagem/espécie de Trichogramma por gerações sucessivas em ovos de $G$. aurantianum, visando a uma possível "adaptação" do parasitóide, poderá levar a maiores percentuais de parasitismo.

O número de adultos emergidos por ovo foi de 1,8 para as linhagens G11 e Br10, as quais diferiram significativamente apenas da linhagem G3, a qual apresentou um número inferior de adultos $(1,3)$ emergidos por ovo (Tabela II). As demais linhagens não diferiram estatisticamente destas três mencionadas.

O número de parasitóides por ovo é variável em função do volume do ovo do hospedeiro. Segundo Vinson (1997), o tamanho do hospedeiro não influencia apenas no número de ovos depositados, mas também no tamanho do adulto de Trichogramma, o qual depende dos recursos nutricionais disponíveis para o desenvolvimento da larva. Assim, há um número ideal de ovos para determinado tamanho de hospedeiro.

Para a longevidade de fêmeas foram encontrados valores distintos apenas para as linhagens Tp e L2, ou seja, 6,3 e 9,3 dias, respectivamente, sendo que as demais linhagens não diferiram destes valores (Tabela II). As longevidades das linhagens/espécies observadas foram superiores àquelas relatadas por outros pesquisadores na mesma faixa de temperatura em diferentes hospedeiros (Calvin et al. 1984; Harrison et al. 1985; Bleicher \& Parra 1989) e inferior aos obtidos por Pratissoli et al. (2004) para cinco linhagens de T. pretiosum criadas em ovos de A. kuehniella (7,3 a 10,9 dias).

Tanto o hospedeiro no qual se desenvolve o parasitóide, quanto a sua origem, podem afetar a longevidade (Bleicher \& Parra 1989; Pratissoli et al. 2004). Entretanto, Stein \& Parra (1987) não encontraram diferenças na longevidade de Trichogramma sp. em ovos de A. kuehniella, Sitotroga cerealella (Olivier, 1819) (Lepidoptera, Gelechiidae) e Plodia interpunctella (Hübner, 1813) (Lepidoptera, Pyralidae).

A razão sexual das linhagens/espécies criadas em ovos de G. aurantianum apresentou valores acima de 0,6 a $25^{\circ} \mathrm{C}$. Os valores foram semelhantes aos obtidos por Pratissoli \& Parra (2001) e Beserra \& Parra (2004), e superiores aos obtidos por Sá \& Parra (1994) em ovos de Helicoverpa zea (Boddie, 1850) (Lepidoptera, Noctuidae) e A. kuehniella $(0,25$ a 0,44$)$ e Pratissoli et al. (2004) em ovos de $S$. cerealella $(0,4$ a 0,6).

Como houve dificuldade para se coletar espécies de Trichogramma em Citrus, e os resultados encontrados por Garcia (1998) foram promissores no controle de G. aurantianum utilizando-se uma linhagem de $T$. pretiosum coletada em outra cultura, optou-se por avaliar as linhagens mantidas pelo Laboratório de Biologia de Insetos da ESALQ/USP e seu potencial de controle do bicho-furão-dos-citros. Neste sentido, avaliaram-se, principalmente, o número de ovos parasitados e a porcentagem de parasitismo, já que o objetivo é controlar a praga com liberações inundativas de Trichogramma dentro de um programa de Controle Biológico Aplicado.

Assim, com base nos parâmetros biológicos avaliados, principalmente no número médio de ovos parasitados e na porcentagem de parasitismo, as linhagens/espécies Atp ( $T$. atopovirilia) e G18 (T. pretiosum) foram selecionadas para os estudos de biologia em ovos de G. aurantianum.

É fundamental, pelos resultados obtidos, que sejam realizadas coletas e seleção de linhagens/espécies mais agressivas à praga, pois houve variação no material biológico estudado que não foi coletado sobre ovos de G. aurantianum.

Número ideal de parasitóides por ovo de G aurantianum. Pela avaliação dos resultados obtidos nos experimentos com T. atopovirilia (linhagem Atp) e T. pretiosum (linhagem G18), não ficou bem caracterizada a espécie com maior potencial para o controle do bicho-furão-dos-citros. Talvez, em testes de campo possa ser definida qual a espécie mais adequada ou 


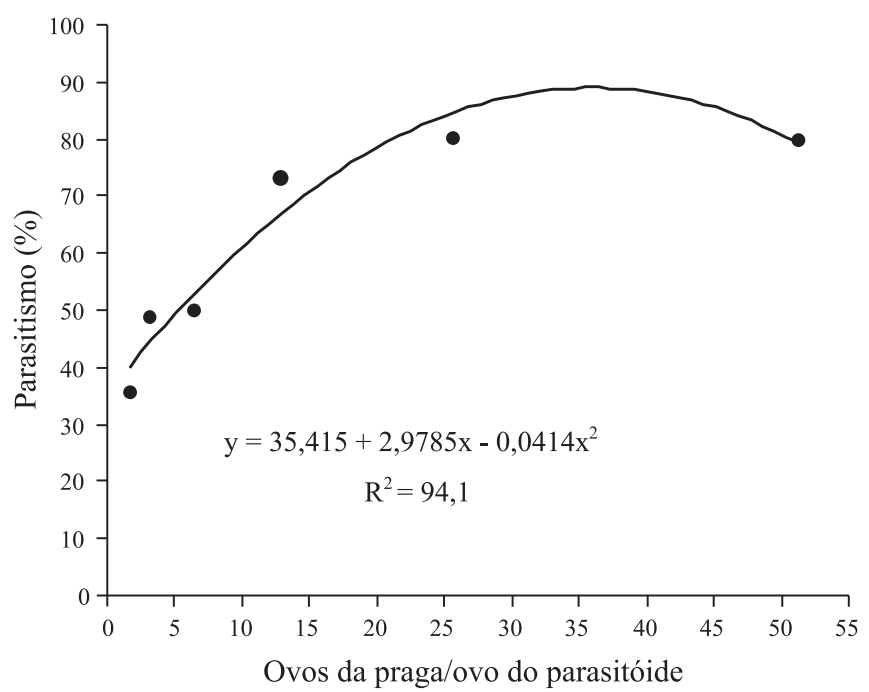

Fig. 1. Relação entre números variáveis de parasitóides (Trichogramma pretiosum - linhagem G18), liberados por ovo (1) de Gymnandrosoma aurantianum e, porcentagem de parasitismo, em dois experimentos, realizados em telado.

até demonstrado que ambas as espécies podem ser utilizadas conjuntamente. Isto porque, no estudo de biologia, $T$. atopovirilia apresentou algumas vantagens, como assincronismo na emergência e maior longevidade de fêmeas em baixa e alta temperaturas $\left(20\right.$ e $\left.32^{\circ} \mathrm{C}\right)$. Segundo Hassan (1997), a combinação de mais de uma espécie de Trichogramma, em liberações desse parasitóide, pode aumentar a sua eficiência no controle de uma praga. A escolha para essa combinação deve ser baseada nos resultados de experimentos de laboratório, telado e campo, e que uma vantagem dessa prática pode ser a de que as duas espécies apresentem aspectos complementares, relacionados ao tempo de emergência, ao comportamento de procura, à longevidade e à tolerância às condições ambientais extremas, características observadas na presente pesquisa.

Apesar destas possibilidades, no experimento de telado optou-se por avaliar $T$. pretiosum, pela disponibilidade de insetos e pela facilidade de obtenção de parasitóides desta espécie, tanto em coletas de campo, como em criações de laboratório.

O percentual de parasitismo de T. pretiosum (linhagem G18) apresentou comportamento quadrático com o aumento da quantidade de parasitóides liberados por ovo da praga, sendo que o ponto máximo de parasitismo estimado foi de $89,0 \%$, o que seria obtido com uma proporção estimada de 36 parasitóides por ovo de G. aurantianum (Fig. 1). Observou-se que a partir deste ponto haveria um decréscimo do parasitismo, o que poderia ser devido à ocorrência de superparasitismo. Knipling (1977) constatou, por meio de modelos de simulação, que números crescentes de parasitóides liberados por unidade de área levam a uma redução na eficiência de Trichogramma, pois, à medida que cresce a sua densidade, a probabilidade de um indivíduo encontrar um ovo diminui. Desta forma, a competição intra-específica torna bastante improvável a ocorrência de um parasitismo próximo a 100\%, em condições de campo.

Garcia (1998) obteve correlação linear positiva entre o número de parasitóides liberados e o percentual de parasitismo para T. pretiosum por ovo de G. aurantianum. Os percentuais de parasitismo variaram de 9,2 a 63,3\% para relações de fêmeas do parasitóide: ovo da praga, de 0,8 a 12,8 , respectivamente.

Pelo resultado obtido no presente trabalho, fica demonstrado que poderão ser necessários números bastante elevados de T. pretiosum, em liberações em áreas de Citrus, para o controle de G. aurantianum, pois se a população de ovos for alta, serão necessárias números elevados de $T$. pretiosum para atender à proporção encontrada de 36 parasitóides por ovo. Para citros e outras frutíferas são recomendadas liberações variáveis de 70.000 a 3,8 milhões/ha de parasitóides ou de 9.000 a 50.000/planta, obtendo-se porcentagens de parasitismo entre 51,8 e $75 \%$ (Oatman \& Platner 1985; Hassan et al. 1988; Newton \& Odendaal 1990; Glen \& Hoffmann 1997; Mills et al. 2000). Entretanto, apesar dos poucos exemplos de sucessos de uso prático de Trichogramma em pomares, em testes de campo têm-se observado resultados promissores.

Assim, tornam-se necessários estudos para avaliar a eficiência de Trichogramma em pomares de citros (condições de campo), pois os resultados até aqui alcançados em telado (semi-campo) demonstram o potencial de utilização desse inimigo natural para controle de G. aurantianum. Os dados obtidos em condições controladas, portanto sem a ação de outros parasitóides e predadores, poderiam ser diferentes daqueles obtidos em campo, o que demandaria, portanto, validação dos resultados obtidos em condições naturais. Entretanto, em geral, as proporções de parasitóides por ovo da praga obtidos em telados, têm sido bastante próximos àquelas obtidas em campo (Parra \& Zucchi 2004). Desta forma, recomenda-se tal proporção para controle de G. aurantianum, aliando-se assim a utilização de feromônio do bicho-furão para determinar o nível de controle da praga (Parra et al. 2004), a partir do qual será liberado o parasitóide referido no presente estudo.

Agradecimentos. Ao Fundo de Defesa da Citricultura FUNDECITRUS, pelo suporte financeiro da pesquisa, à Dra ${ }^{\mathrm{a}}$. Ranyse B. Querino, pela identificação das espécies de Trichogramma.

\section{REFERÊNCIAS}

Beserra, E. B. \& J. R. P. Parra. 2004. Biologia e parasitismo de Trichogramma atopovirilia Oatman \& Platner e Trichogramma pretiosum Riley (Hymenoptera, Trichogrammatidae) em ovos de Spodoptera frugiperda (J. E. Smith) (Lepidoptera, Noctuidae). Revista Brasileira de Entomologia 48: 119-126.

Bleicher, E. \& J. R. P. Parra. 1989. Espécies de Trichogramma parasitóides de Alabama argillacea. I. Biologia de três populações. Pesquisa Agropecuária Brasileira 24: 929-940.

Calvin, D. D.; M. C. Knapp; S. M. Welch; F. L. Poston \& R. Elzinga 1984. Impact of environmental factors on Trichogramma pretiosum reared on southwestern corn borer eggs. Environmental Entomology 13: 774-780. 
Fundecitrus. 2000. Tecnologia contra o bicho-furão. Revista do Fundecitrus 96: 8-10.

Garcia, M. S. 1998. Bioecologia e potencial de controle biológico de Ecdytolopha aurantiana (Lima, 1927) (Lepidoptera: Tortricidae), o bicho-furão-dos-citros, através de Trichogramma pretiosum Riley, 1879. Piracicaba. Tese de doutorado. Escola Superior de Agricultura "Luiz de Queiroz", USP, $118 \mathrm{p}$.

Garcia, M. S. \& J. R. P. Parra. 1999. Comparação de dietas artificiais, com fontes protéicas variáveis, para criação de Ecdytolopha aurantiana (Lima, 1927) (Lepidoptera: Tortricidae). Anais da Sociedade Entomológica do Brasil 28: 219-232.

Glenn, D. C. \& A. A. Hoffmann. 1997. Developing a commercially viable system for biological control of light brown apple moth (Lepidoptera: Tortricidae) in grapes using endemic Trichogramma (Hymenoptera: Trichogrammatidae). Journal of Economic Entomology 90: 370-382.

Harrison, W. W.; E. G. King \& J. D. Ouzts. 1985. Development of Trichogramma exiguum and $T$. pretiosum at five temperature regimes. Environmental Entomology 14: 118-121.

Hassan, S. A. 1989. Selection of suitable Trichogramma strains to control the codling moth Cydia pomonella and the two summer fruit tortrix moths Adoxophyes orana, Pandemis heparana (Lep.: Tortricidae). Entomophaga 34: 19-27.

Hassan, S. A. 1997. Seleção de espécies de Trichogramma para o uso em programas de controle biológico, p.183-205. In: Parra, J. R. P. \& Zucchi, R. A. (eds.). Trichogramma e o controle biológico aplicado. Piracicaba, FEALQ, vii+324 p.

Hassan, S. A. \& M. F. Guo. 1991. Selection of effective strains of egg parasites of the genus Trichogramma (Hym., Trichogrammatidae) to control the european corn borer Ostrinia nubilalis Hübner (Lep., Pyralidae). Journal of Applied Entomology 111: 335-341.

Hassan, S. A.; E. Kolher \& W. M. Rost. 1988. Mass production and utilization of Trichogramma: 1. Control of the codling moth, Cydia pomonella and the summer fruit tortrix moth Adoxophyes orana (Lep.: Tortricidae). Entomophaga 33: 413-420.

Knipling, E. F. 1977. The theoretical basis for augmentation of natural enemies. p. 79-123. In: Ridgway, R. L. \& S. B. Vinson (eds.). Biological control by augmentation of natural enemies. New York, Plenum Press, iii+480 p.

Li, S. Y.; D. E. Henderson \& J. H. Myers. 1994. Selection of suitable Trichogramma species for potential control of the blackheaded fireworm infesting cranberries. Biological Control 4: 244-248.

Mills, N.; C. Pickel; S. Mansfield; S. Mcdougall; R. Buchner; J. Caprile; J. Edstrom; R. Elkins; J. Hasey; K. Kelley; B. Krueger; B. Olson \& R. Stocker. 2000. Mass releases of Trichogramma wasps can reduce damage from codling moth. California Agriculture 56: 22-25.

Newton, P. J. \& W. J. Odendaal. 1990. Commercial inundative releases of Trichogrammatoidea cryptophlebiae (Hym.: Trichogrammatidae) against Cryptophlebia leucotreta (Lep.:
Tortricidae) in Citrus. Entomophaga 35: 545-556.

Oatman, E. R. \& G. R. Platner. 1985. Biological control of two avocado pests. California Agriculture 39: 21-23.

Parra, J. R. P. 1997. Técnicas de criação de Anagasta kuehniella, hospedeiro alternativo para produção de Trichogramma, p. 121150. In: Parra, J. R. P. \& R. A. Zucchi (eds.). Trichogramma e o controle biológico aplicado. Piracicaba, FEALQ, iv+324 p.

Parra, J. R. P.; J. M. S. Bento; M. S. Garcia; P. T. Yamamoto; E. F. Vilela \& W. S. Leal. 2004. Development of a control alternative for the citrus fruit borer, Ecdytolopha aurantiana (Lepidoptera, Tortricidae): from basic research to the grower. Revista Brasileira de Entomologia 48: 561-567.

Parra, J. R. P. \& R. A. Zucchi. 2004. Trichogramma in Brazil: Feasibility of use after twenty years of research. Neotropical Entomology 33: $271-281$.

Pratissoli, D. \& J. R. P. Parra. 2000. Desenvolvimento e exigências térmicas de Trichogramma pretiosum Riley, criados em duas traças do tomateiro. Pesquisa Agropecuária Brasileira 35: 12811288.

Pratissoli, D. \& J. R. P. Parra. 2001. Seleção de linhagens de Trichogramma pretiosum Riley (Hymenoptera: Trichogrammatidae) para o controle das traças Tuta absoluta (Meyrick) e Phthorimaea operculella (Zeller) (Lepidoptera: Gelechiidae). Neotropical Entomology 30: 277-282.

Pratissoli, D.; A. M. Holtz; J. R. Gonçalves; R. C. Oliveira \& U. R. Vianna. 2004. Características biológicas de linhagens de Trichogramma pretiosum, criados em ovos de Sitotroga cerealella e Anagasta kuehniella. Horticultura Brasileira 22: 562-565.

Sá, L. A. N. \& Parra J. R. P. 1994. Biology and parasitism of Trichogramma pretiosum Riley (Hym., Trichogrammatidae) on Ephestia kuehniella (Zeller) (Lep., Pyralidae) and Heliothis zea (Boddie) (Lep., Noctuidae) eggs. Journal of Applied Entomology 118: $38-43$.

Sas Institute. 1999. SAS System - SAS/STAT software: version 8.0, Cary.

Stein, C. P. \& J. R. P. Parra. 1987. Aspectos biológicos de Trichogramma sp. em diferentes hospedeiros. Anais da Sociedade Entomológica do Brasil 16: 163-169.

Torres P. C. \& M. Gerding P. 2000. Evaluación de cinco especies de Trichogramma como posibles agentes de control biológico de Cydia pomonella (L.) (Lepidoptera: Tortricidae). Agricultura Técnica 60: $282-288$.

Wührer, B. G. \& S. A. Hassan. 1993. Selection of effective species/ strains of Trichogramma (Hym., Trichogrammatidae) to control the diamondback moth Plutella xylostella L. (Lep., Plutellidae). Journal of Applied Entomology 116: 80-89.

Vinson, S. B. 1997. Comportamento de seleção hospedeira de parasitóides de ovos, com ênfase na família Trichogrammatidae, p. 67-119. In: Parra, J. R. P. \& R. A. Zucchi, (eds.). Trichogramma e o controle biológico aplicado. Piracicaba, FEALQ, iii+324 p. 